\section{EAACI-Auszeichnung geht nach Athen}

D er Allergopharma Award wurde im Jahre 2000 in Kooperation mit der European Academy of Allergology and Clinical Immunology (EAACI) ins Leben gerufen. Das Ziel des mit 10.000 Euro dotierten Preises ist es, herausragende Forschungsarbeiten jüngerer Mitglieder der Akademie auf dem Gebiet der Mechanismen allergischer Entzündungen sowie der allergenspezifischen Immuntherapie zu würdigen. Der diesjährige 8. Award wurde während des 27. Kongresses der Akademie Anfang Juni in Barcelona verliehen: EAACI-Präsident Prof. Dr. Roy Gerth van Wijk zeichnete Dr. Georgina Xanthou-Tsingoglou, Athen, Griechenland, aus für ihre Arbeit „Osteopontin has a crucial role in allergic airway disease through regulation of dendritic cell subsets" (Nat Med 2007; 13: 570-8).

Dr. Xanthou-Tsingoglou promovierte an der Universität von Athen. In ihrer Doktorarbeit befasste sie sich mit der Rolle der Chemokine, die beim Sjögren-Syndrom von antigenpräsentierenden Zellen gebildet werden. Auch bei ihren folgenden Arbeiten am Imperial College in London waren die Chemokine ein zentrales Thema. Hier untersuchte sie funktionelle Wechselwirkungen zwischen Chemokinrezeptoren und deren Einfluss auf Lymphozyten-Subpopulationen während einer Immunantwort. Schließlich kehrte sie nach Athen zurück,

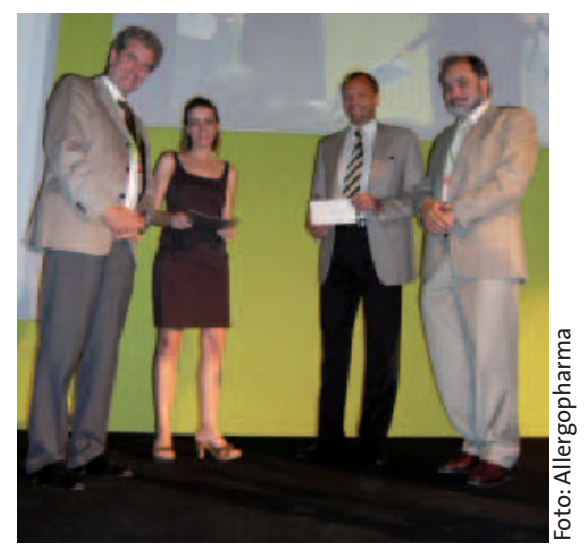

Von links nach rechts: Prof. Dr. Ulrich Wahn, Preisträgerin Dr. Georgina Xanthou-Tsingoglou, Lars Ingemann, Allergopharma, und EAACI-Präsident Dr. Roy Gerth van Wijk

um eine Anstellung als Assistenzprofessorin bei der Biomedical Research Foundation anzunehmen.

Aktuell beschäftigt sich Xanthou speziell mit den Mediatoren Osteopontin und Activin-A und deren Rolle bei der Induktion und Regulation von TZellen bei Autoimmunerkrankungen und Allergien. In ihrer jetzt prämierten Publikation in Nature Medicine konnte sie zeigen, dass Osteopontin durch Regulation von T-Helferzellen und Subpopulationen von dendritischen Zellen eine wichtige Rolle bei der Allergie spielt.

\title{
Ekzempatienten unterversorgt
}

A $\mathrm{m}$ 3. Juli 2008 diskutierten in Brüssel Vertreter der Europäischen Akademie für Dermatologie und Venerologie (EADV) mit Patientenorganisationen und EU-Offiziellen zur Problematik der Versorgung und Betreuung von Patienten mit atopischer Dermatitis in Europa. Es wurde das deutsche Programm der "Neurodermitisschulung" vorgestellt, deren Wirksamkeit erwiesen ist, die jedoch nur wenige Betroffene erreicht.

Die Einschränkung der Lebensqualität - durch Juckreiz, Entstellung und

gestörte Hautfunktion - ist bei Ekzemerkrankungen erheblich und stellt bei einer Häufigkeit von 10-20\% Betroffener in der Bevölkerung ein großes sozioökonomisches Problem dar. Prof. Dr. Dr. Johannes Ring, München, sprach von einem „Skandal“, weil viele Neurodermitiskranke die Basisbehandlung mit Cremes selbst bezahlen müssten, das wäre wie „wenn Diabetiker für das Insulin selbst aufkommen müssten"! Die EADV will im Rahmen einer „Ekzemkampagne“ in Zukunft verstärkt auf diese Missstände hinweisen.

\section{Deutscher Chef für WAO-Journal}

Die World Allergy Organization (WAO) hat Prof. Dr. Dr. Johannes Ring, Klinik und Poliklinik für Dermatologie und Allergologie am Biederstein, TU München, zum Editor-in-Chief des WAO-Journals bestimmt. Die Zweitschrift erscheint seit Anfang des Jahres 2008 und wird an Interessierte und Mitglieder der nationalen allergologischen Gesellschaften kostenlos elektronisch zugestellt. Damit erreicht das WAO-Journal über 30.000 allergologisch tätige Ärzte weltweit. Weitere Informationen bietet unter www.waojournal.org das Internet.

\section{Lungentag ante portas}

Am 27. September ist es wieder soweit: Im Rahmen des Lungentages soll das Bewusstsein über Atemwegs- und Lungenerkrankungen in Deutschland geschärft werden. Im letzten Jahr feierte der Lungentag bereits sein zehnjähriges Bestehen und konnte rund 50.000 Besucher begrüßen, die neben den zahlreichen Informationsveranstaltungen auch die Möglichkeit zur Lungentestung nutzten. Es wurden circa 6.200 Lungenfunktionsprüfungen durchgeführt, davon ergaben $44 \%$ einen auffälligen Befund, so dass zur Kontrolle beim Facharzt geraten wurde. Der Lungentag 2008 steht unter dem Motto „Reine Luft Gesunde Lunge“. Dabei soll besonders über das Zigarettenrauchen und andere Umweltschadstoffe aufgeklärt werden. Weitere Informationen sowie eine Übersicht über die mehr als 200 Veranstaltungen in diesem Jahr sind unter www.lungentag.de abzurufen.

\section{Finanzspritze für Filaggrin-Forschung}

Priv.-Doz. Dr. Stephan Weidinger und Priv.-Doz. Dr. Martin Mempel, beide von der Klinik und Poliklinik für Dermatologie und Allergologie am Biederstein, TU München, und vom ZAUM - Zentrum für Allergie und Umwelt, haben den „Research on skin-dryness award“ (ROSA) des Laboratoriums Roche-Posay erhalten. Der Preis war ausgeschrieben zur Finanzierung eines wegweisenden Forschungsprojekts, die beiden Münchner Wissenschaftler haben mit „Die Rolle der durch Filaggrin-Defizienz bedingten epidermalen Barrierestörung für die IgESensibilisierung " das interessanteste Konzept vorgelegt. 